\title{
A Description of the MISTY1 Encryption Algorithm
}

Status of this Memo

This memo provides information for the Internet community. It does not specify an Internet standard of any kind. Distribution of this memo is unlimited.

Copyright Notice

Copyright (C) The Internet Society (2000). All Rights Reserved.

Abstract

This document describes a secret-key cryptosystem MISTY1, which is block cipher with a 128-bit key, a 64-bit block and a variable number of rounds. It documents the algorithm description including key scheduling part and data randomizing part.

1. Introduction

This document describes a secret-key cryptosystem MISTY1, which is block cipher with a 128-bit key, a 64-bit block and a variable number of rounds. It is designed on the basis of the theory of provable security against differential and linear cryptanalysis, and moreover it realizes high-speed encryption on hardware platforms as well as on software environments. As the result of weighing strength and speed, 8-rounds of MISTY1 is recommended and used in most cases.

Our implementation shows that MISTY1 with eight rounds can encrypt a data stream in CBC mode at a speed of 57Mbps and 40Mbps on Pentium $\mathrm{II} / 266 \mathrm{MHz}$ and $\mathrm{PA}-7200 / 120 \mathrm{MHz}$, respectively. For its hardware performance, we have produced a prototype LSI by a process of $0.8-$ micron CMOS gate-array and confirmed a speed of 512Mbps.

2. Algorithm Description

Algorithm [1] could be divided into two parts, namely "key scheduling part" and "data randomizing part". Key scheduling part takes a 128bit input key and produces a 128-bit expanded key. Data randomizing 
part takes a 64-bit input data and mixes it, namely encryption. If data randomizing part is processed in reverse order, mixed data is transformed to input data, namely decryption.

\subsection{Terminology}

Some operators are used in this document to describe the algorithm. The operator ' + ' indicates two's complement addition. The operator '*' indicates multiplication. The operator '/' yields the quotient, and the operator '' yields the remainder from the division. The operator ' $\&$ ' indicates bitwise AND operation. The operator '|' indicates bitwise inclusive OR operation. The operator '^' indicates bitwise exclusive OR operation. The operator ' $<$ ' indicates bitwise left shift operation. The operator ' $>>$ ' indicates bitwise right shift operation.

\subsection{Key Scheduling Part}

Key scheduling part consists of the following operations.

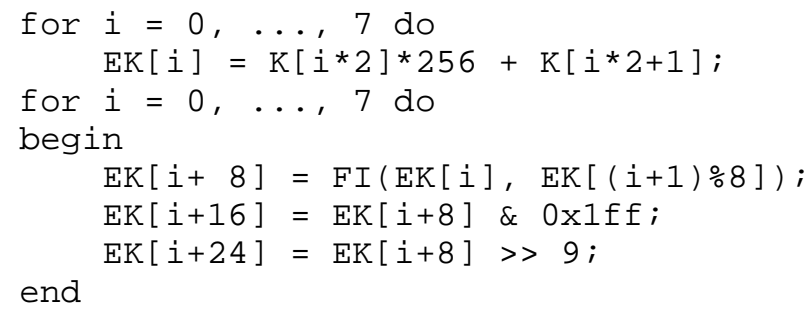

$K$ is an input key, and each element of $K$, namely $K[i]$, holds an 8-bit of the key, respectively. EK denotes an expanded key, and each element of EK, namely EK[i], holds a 16-bit of the expanded key. Input data of K[0], ..., K[15] are copied to EK[0], ..., EK[7]. Expanded key is produced from EK[0], ..., EK[7] by using function FI, and stored in EK[8], ..., EK[15]. Function FI is described in the following section.

\subsection{Data Randomizing Part}

Data randomizing part uses two kinds of function, which are called function FO and function FL. Function FO calls another function, namely FI. The key expansion part also uses function FI. Function FI uses two S-boxes, namely S7, S9. Each function is described as follows.

Function FO takes two parameters. One is a 32-bit width input data, namely FO_IN. The other is an index of EK, namely k. And FO returns a 32-bit width data, namely FO_OUT. 


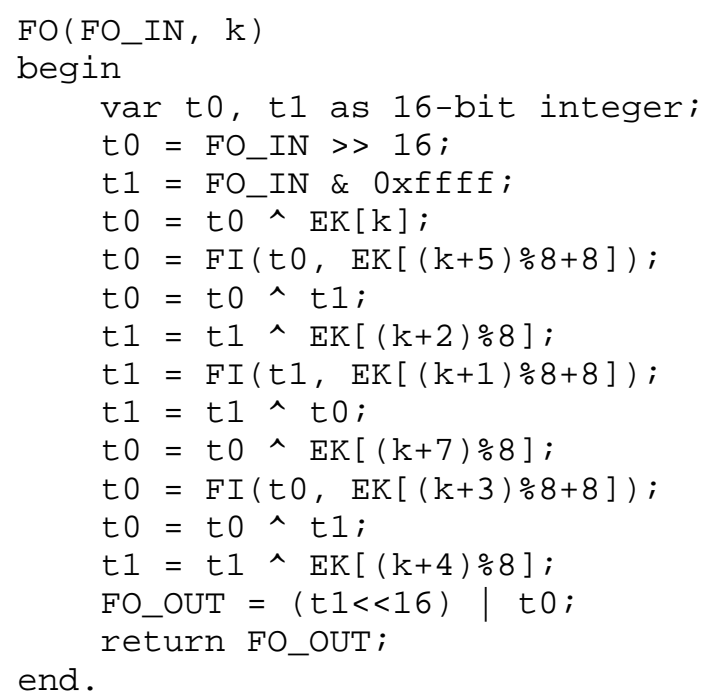

Function FI takes two parameters. One is a 16-bit width input data, namely FI_IN. The other is a part of EK, namely FI_KEY, which is also 16-bit width. And FI returns a 16-bit width data, namely FI_OUT.

FI (FI_IN, FI_KEY)

begin

var d9 as 9-bit integer;

var d7 as 7-bit integer;

$\mathrm{d} 9=$ FI_IN $>7$;

$\mathrm{d} 7=$ FI_IN \& $0 \times 7 f$;

$d 9=$ S9TABLE $[d 9] \wedge d 7 ;$

$d 7=$ S7TABLE[d7] ^ $d 9$;

$(d 7=d 7 \& 0 \times 7 f ;)$

$\mathrm{d} 7=\mathrm{d} 7 \wedge$ (FI_KEY $>>$ 9);

$\mathrm{d} 9=\mathrm{d} 9 \wedge(\mathrm{FI} K \mathrm{KEY} \& 0 \times 1 \mathrm{ff}) ;$

d9 $=$ S9TABLE $[\mathrm{d} 9] \wedge d 7$;

FI_OUT $=(d 7<<9) \quad$ d9;

return FI_OUT;

end.

S7TABLE and S9TABLE denote the S-boxes $S 7$ and $S 9$ respectively in terms of look up table notation. Here are the description of S7TABLE and S9TABLE in hexadecimal notation. 


\begin{tabular}{|c|c|c|c|c|c|c|c|c|c|c|c|c|c|c|c|c|}
\hline \multicolumn{17}{|c|}{$7 \mathrm{TAB}$} \\
\hline & 0 & 1 & 2 & 3 & 4 & 5 & 6 & 7 & 8 & 9 & a & $\mathrm{b}$ & C & $d$ & e & f \\
\hline . & $1 \mathrm{~b}$ & 32 & 33 & $5 a$ & $3 b$ & 10 & 17 & 54 & $5 b$ & $1 a$ & 72 & 73 & $6 \mathrm{~b}$ & $2 c$ & 66 & \\
\hline 10 : & $1 \mathrm{f}$ & 24 & 13 & $6 c$ & 37 & $2 e$ & $3 f$ & $4 a$ & $5 d$ & $0 f$ & 40 & 56 & 25 & 51 & $1 \mathrm{C}$ & 0 \\
\hline 0 & $0 \mathrm{~b}$ & 46 & 20 & $0 \mathrm{~d}$ & $7 \mathrm{~b}$ & 35 & 44 & 42 & $2 \mathrm{~b}$ & $1 e$ & 41 & 14 & $4 \mathrm{~b}$ & 79 & 15 & \\
\hline 0 & $0 e$ & 55 & 09 & 36 & 74 & $0 \mathrm{C}$ & 67 & 53 & 28 & $0 a$ & $7 e$ & 38 & 02 & 07 & 60 & \\
\hline 40 & 19 & 12 & 65 & $2 \mathrm{f}$ & 30 & 39 & 08 & 68 & $5 f$ & 78 & $2 a$ & $4 \mathrm{C}$ & 64 & 45 & 75 & \\
\hline 0 & 59 & 48 & 03 & 57 & $7 \mathrm{C}$ & $4 \mathrm{f}$ & 62 & $3 c$ & $1 d$ & 21 & $5 e$ & 27 & $6 a$ & 70 & $4 d$ & \\
\hline & 01 & $6 \mathrm{~d}$ & $6 e$ & 63 & 18 & 77 & 23 & 05 & 26 & 76 & 00 & 31 & $2 d$ & $7 a$ & $7 \mathrm{f}$ & \\
\hline 70 & 50 & 22 & 11 & 06 & 47 & 16 & 52 & $4 e$ & 71 & $3 e$ & 69 & 43 & 34 & $5 c$ & 58 & \\
\hline
\end{tabular}

\section{S9TABLE :}

$\begin{array}{lllllllllllllllll}0 & 1 & 2 & 3 & 4 & 5 & 6 & 7 & 8 & 9 & \mathrm{a} & \mathrm{b} & \mathrm{c} & \mathrm{d} & \mathrm{e} & \mathrm{f}\end{array}$ 000: 1c3 0cb 153 19f le3 0 e9 $0 \mathrm{fb} 035181$ 0b9 117 1eb $13300902 \mathrm{~d} \quad 0 \mathrm{~d} 3$ 010: 0c7 14a 037 07e 0eb $164 \quad 193$ 1d8 0a3 11e 055 02c 01d 1a2 163118 020: 14b 152 1d2 00f 02b 030 13a 0 e5 $111 \quad 138 \quad 18$ e 063 0e3 0 c8 $1 \mathrm{f} 4$ 01b 030: 001 09d 0f8 1a0 16d 1f3 01c 146 07d 0d1 082 1ea 183 12d 0f4 19e 040: 1d3 0dd 1e2 128 le0 0 ec $059091011 \quad 12 \mathrm{f} 026 \quad 0 \mathrm{dc} 0 \mathrm{~b} 0$ 18c $10 \mathrm{f} 1 \mathrm{f}$ 050: 0e7 16c 0b6 0f9 $0 d 8$ 151 101 14c 103 0b8 154 12b 1 ae $017 \quad 071 \quad 00 \mathrm{c}$ 060: 047058 07f 1a4 134129084 15d 19d 1b2 1a3 048 07c 051 1ca 023 070: 13d 1a7 165 03b 042 0da 192 0ce 0c1 06b $09 f$ 1fl 12c $1840 \mathrm{fa} 196$

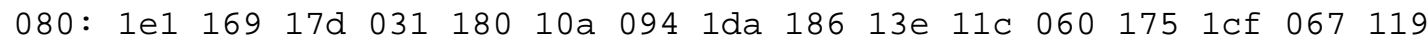

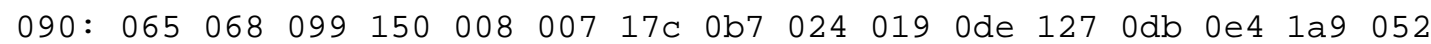
0a0: 109090 19c 1c1 028 1b3 135 16a 176 0df 1 e5 188 0c5 16e 1 de 1 b1 0b0: 0c3 1df 036 0ee 1ee 0f0 $09304909 a \quad 1 b 6069081 \quad 12500 b \quad 05 e$ 0b4 0c0: 149 1c7 174 03e 13b 1b7 08e 1c6 0ae 010095 lef $04 \mathrm{e} 0 \mathrm{f} 2$ 1fd 085

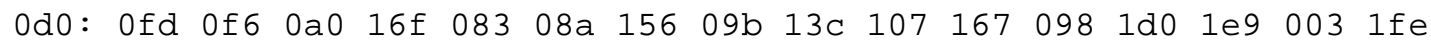
0e0: 0bd 122089 0d2 18f 012033 06a 142 0ed 170 11b 0e2 $14 \mathrm{f} 158131$

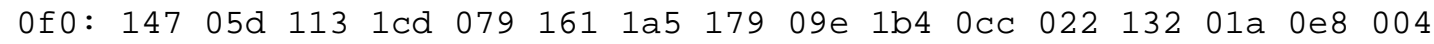

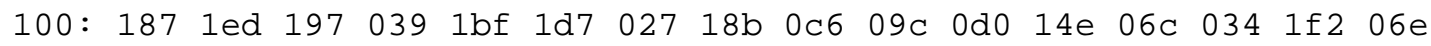
110: Oca 025 0ba 191 Ofe 013106 02f $1 \mathrm{ad} 172 \quad 1 \mathrm{db}$ 0c0 10b $1 \mathrm{~d} 6$ of5 $1 \mathrm{ec}$ 120: 10d 076114 lab 075 10c 1e4 159054 11f $04 \mathrm{~b}$ 0c4 1be $0 f 70290 \mathrm{a} 4$ 130: 00e 1f0 077 04d 17a 086 08b 0 b3 171 Obf 10 e 104097 15b 160168 140: 0d7 0bb 066 1ce 0fc 092 1c5 $06 \mathrm{f} 016$ 04a $0 \mathrm{al} 139$ 0af $0 \mathrm{fl} 190$ 00a

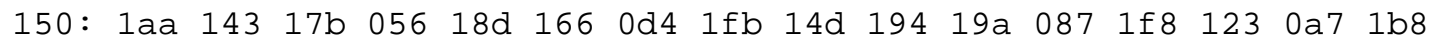
160: 141 03c $1 \mathrm{fg} 140$ 02a 155 11a $1 \mathrm{a} 1 \quad 198$ 0d5 126 laf 061 12e 157 1dc 170: 072 18a 0aa 096115 0ef 045 07b $08 d \quad 145053$ 05f 178 0b2 02 e 020 180: 1d5 03f 1c9 1e7 lac 044038 014 0b1 16b 0ab 0b5 05a 182 1c8 1d4

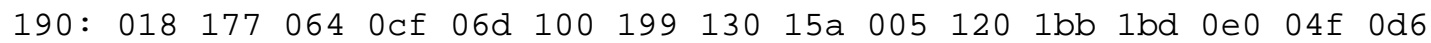
la0: 13f 1c4 12a 015006 off $19 \mathrm{~b} 0 \mathrm{a} 6 \quad 043088050 \quad 15 \mathrm{f} 1 \mathrm{e} 8121073 \quad 17 \mathrm{e}$ 1b0: 0bc 0c2 0c9 173189 1f5 074 1cc 1e6 la8 $19501 \mathrm{f} 04100 \mathrm{~d}$ 1ba 032

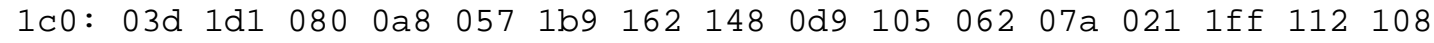
1d0: 1c0 0a9 11d 1b0 1a6 0cd 0f3 05c 102 05b 1d9 144 1f6 0ad 0a5 03a 1e0: $1 \mathrm{cb} 13617 \mathrm{f} 046$ oel $01 \mathrm{e} 1 \mathrm{dd} 0 \mathrm{e} 6137$ fa $18508 \mathrm{c} 08 \mathrm{f} 040$ 1b5 0be 1f0: 078000 0ac $11015 \mathrm{e} 124002$ 1bc 0a2 0ea 070 1fc 116 15c 04c 1c2 
Function FL takes two parameters. One is a 32-bit data, namely FL_IN. The other is an index of EK, namely k. And FL returns a 32bit width data, namely FL_OUT.

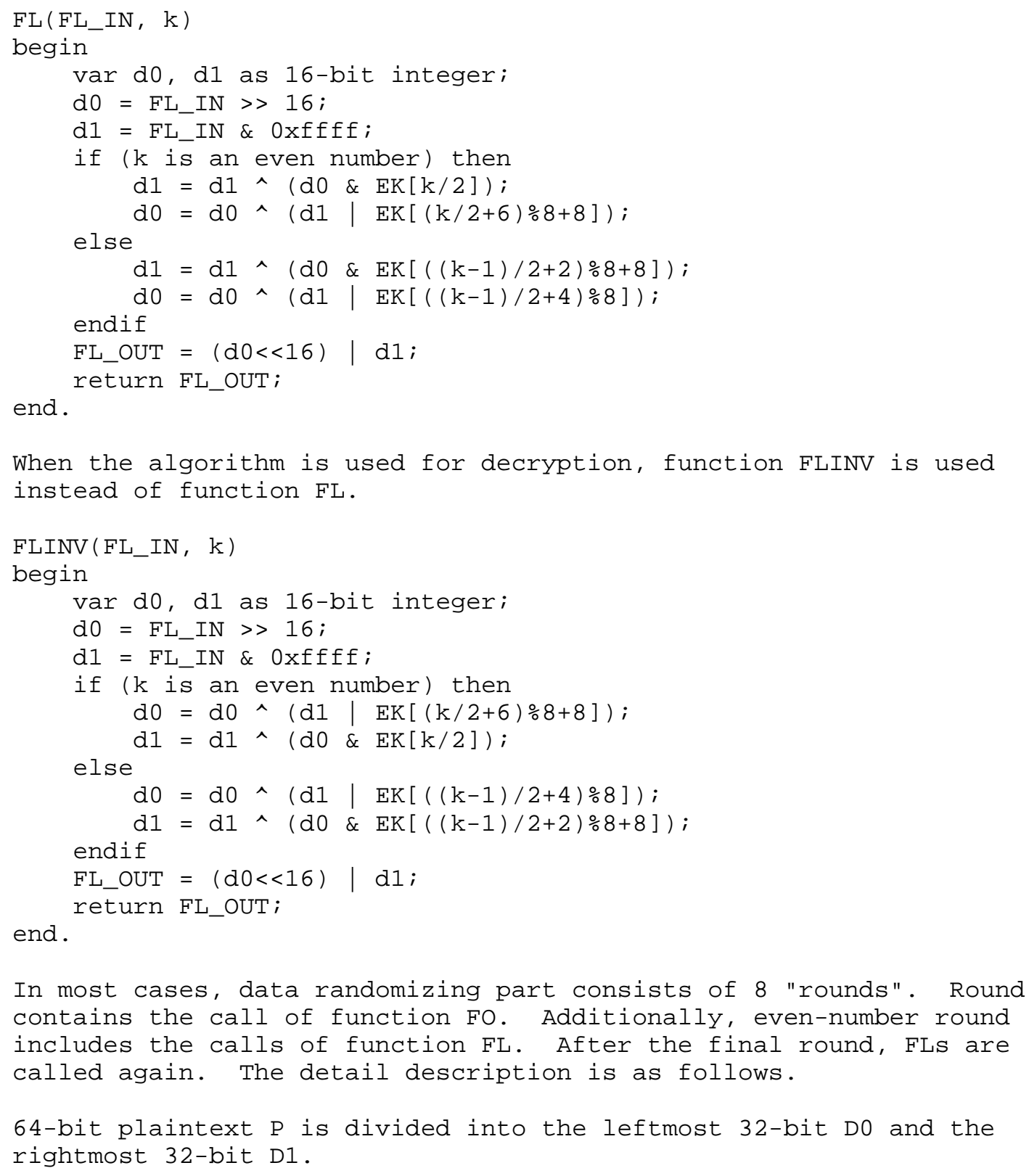


// 0 round

$\mathrm{D} 0=\mathrm{FL}(\mathrm{D} 0,0)$;

$\mathrm{D} 1=\mathrm{FL}(\mathrm{D} 1,1)$;

$\mathrm{D} 1=\mathrm{D} 1 \wedge \mathrm{FO}(\mathrm{D} 0,0)$;

// 1 round

$\mathrm{D} 0=\mathrm{DO} \wedge \mathrm{FO}(\mathrm{D} 1,1)$;

// 2 round

$\mathrm{DO}=\mathrm{FL}(\mathrm{DO}, 2)$;

$\mathrm{D} 1=\mathrm{FL}(\mathrm{D} 1,3)$;

$\mathrm{D} 1=\mathrm{D} 1 \wedge \mathrm{FO}(\mathrm{D} 0,2)$;

// 3 round

$\mathrm{D} 0=\mathrm{D} 0 \wedge \mathrm{FO}(\mathrm{D} 1,3)$;

// 4 round

$\mathrm{DO}=\mathrm{FL}(\mathrm{D} 0,4)$;

$\mathrm{D} 1=\mathrm{FL}(\mathrm{D} 1,5)$;

$\mathrm{D} 1=\mathrm{D} 1 \wedge \mathrm{FO}(\mathrm{D} 0,4)$;

// 5 round

$\mathrm{D} 0=\mathrm{D} 0 \wedge \mathrm{FO}(\mathrm{D} 1,5)$;

// 6 round

$\mathrm{DO}=\mathrm{FL}(\mathrm{D} 0,6)$;

$\mathrm{D} 1=\mathrm{FL}(\mathrm{D} 1,7)$;

$\mathrm{D} 1=\mathrm{D} 1 \wedge \mathrm{FO}(\mathrm{D} 0,6)$;

// 7 round

$\mathrm{D} 0=\mathrm{D} 0 \wedge \mathrm{FO}(\mathrm{D} 1,7)$;

// final

$\mathrm{DO}=\mathrm{FL}(\mathrm{D} 0,8)$;

$\mathrm{D} 1=\mathrm{FL}(\mathrm{D} 1,9) ;$

64-bit ciphertext $\mathrm{C}$ is constructed from DO and D1 as following operation.

$\mathrm{C}=(\mathrm{D} 1<<32) \mid \mathrm{D} 0$;

When data randomizing part is used as decrypting operation, it should be executed in reverse order. The detail description is as follows.

DO $=\mathrm{C} \&$ Oxffffefff;

$\mathrm{D} 1=\mathrm{C}>>32$;

$\mathrm{DO}=\mathrm{FLINV}(\mathrm{D} 0,8) ;$

$\mathrm{D} 1=\mathrm{FLINV}(\mathrm{D} 1,9)$;

$\mathrm{DO}=\mathrm{DO} \wedge \mathrm{FO}(\mathrm{D} 1,7)$;

$\mathrm{D} 1=\mathrm{D} 1 \wedge \mathrm{FO}(\mathrm{D} 0,6)$;

$\mathrm{DO}=\mathrm{FLINV}(\mathrm{D} 0,6)$;

$\mathrm{D} 1=\mathrm{FLINV}(\mathrm{D} 1,7) ;$

$\mathrm{D} 0=\mathrm{D} 0 \wedge \mathrm{FO}(\mathrm{D} 1,5) ;$

$\mathrm{D} 1=\mathrm{D} 1 \wedge \mathrm{FO}(\mathrm{D} 0,4)$;

$\mathrm{DO}=\operatorname{FLINV}(\mathrm{D} 0,4)$;

$\mathrm{D} 1=\operatorname{FLINV}(\mathrm{D} 1,5)$; 


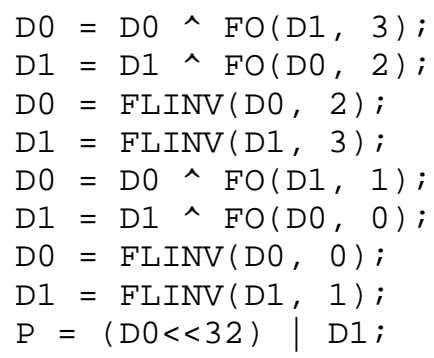

3. Object Identifier

The Object Identifier for MISTY1 in Cipher Block Chaining (CBC) mode is as follows:

MISTY1-CBC OBJECT IDENTIFIER : :=

\{iso(1) member-body (2) jisc(392)

mitsubishi-electric-corporation(200011) isl(61) security(1)

algorithm(1) symmetric-encryption-algorithm(1) misty1-cbc(1) \}

MISTY1-CBC needs Initialization Vector (IV) as like as other

algorithms, such as DES-CBC, DES-EDE3-CBC and so on. To determine

the value of IV, MISTY1-CBC takes parameter as:

MISTY1-CBC Parameter : := IV

where IV ::= OCTET STRING -- 8 octets.

When this Object Identifier is used, plaintext is padded before encrypt it. At least 1 padding octet is appended at the end of the plaintext to make the length of the plaintext to the multiple of 8 octets. The value of these octets is as same as the number of appended octets. (e.g., If 5 octets are needed to pad, the value is 0x05.)

4. Security Considerations

The algorithm, which is described in this document, is designed in consideration of the theory of provable security against differential cryptanalysis and linear cryptanalysis [2] [3] [4]. According to the recent result, when the algorithm consists of 8 rounds, both differential characteristic probability and liner characteristic probability are $2^{\wedge}-140$. For reference, probabilities of DES are $2^{\wedge}-$ 62 and $2^{\wedge}-46$, respectively. 
5. Legal Issues

The algorithm description is applied for a patent in several countries as PCT/JP96/02154. However, the algorithm is freely available for academic (non-profit) use. Additionally, the algorithm can be used for commercial use without paying the patent fee if you contract with Mitsubishi Electric Corporation. For more information, please contact at MISTYeisl.melco.co.jp.

6. References

[1] M. Matsui, "New Block Encryption Algorithm MISTY", Fast Software Encryption - 4th International Workshop (FSE'97), LNCS 1267, Springer Verlag, 1997, pp.54-68

[2] K. Nyberg and L.R. Knudsen, "Provable Security Against a Differential Attack", Journal of Cryptology, Vol.8, No.1, 1995, pp. $27-37$

[3] K. Nyberg, "Linear Approximation of Block Ciphers", Advances in Cryptology - Eurocrypt'94, LNCS 950, Springer Verlag, 1995, pp. 439-444

[4] M. Matsui, "New Structure of Block Ciphers with Provable Security Against Differential and Linear Cryptanalysis", Fast Software Encryption - Third International Workshop, LNCS 1039, Springer Verlag, 1996, pp.205-218

7. Authors' Addresses

Hidenori Ohta Mitsubishi Electric Corporation, Information Technology R\&D Center 5-1-1 Ofuna, Kamakura, Kanagawa 247-8501, Japan

Phone: $+81-467-41-2183$

Fax: $\quad+81-467-41-2185$

EMail: hidenoridiss.isl.melco.co.jp

Mitsuru Matsui

Mitsubishi Electric Corporation, Information Technology R\&D Center 5-1-1 Ofuna, Kamakura, Kanagawa 247-8501, Japan

Phone: +81-467-41-2181

Fax: $\quad+81-467-41-2185$

EMail: matsui@iss.isl.melco.co.jp 
Appendix A. Example Data of MISTY1

Here is an example ciphertext of MISTY1 when the key and the plaintext are set as following value.

Key: $\quad 001122 \quad 3344556677 \quad 8899$ aa bb cc dd ee ff

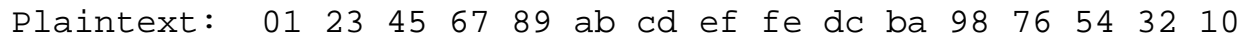

Ciphertext: 8b 1d a5 f5 $6 \mathrm{a}$ b3 d0 7c 04 b6 8240 b1 3b e9 $5 d$

In the above example, because the plaintext has a length of 128-bit, MISTY1 is used two times to each 64-bit, namely ECB mode.

Following example is ciphertext of MISTY1 in CBC mode.

Key: $\quad 00112233445566778899$ aa bb cc dd ee ff

IV: $\quad 010203040506 \quad 0708$

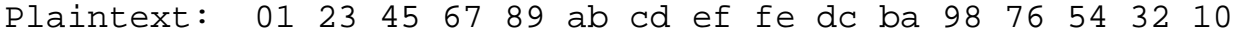

Ciphertext: 46 lc $1 \mathrm{e} 87$ 9c $18 \mathrm{c} 27 \mathrm{f}$ b9 ad f2 d8 0c 8903 if 
Full Copyright statement

Copyright (C) The Internet Society (2000). All Rights Reserved.

This document and translations of it may be copied and furnished to others, and derivative works that comment on or otherwise explain it or assist in its implementation may be prepared, copied, published and distributed, in whole or in part, without restriction of any kind, provided that the above copyright notice and this paragraph are included on all such copies and derivative works. However, this document itself may not be modified in any way, such as by removing the copyright notice or references to the Internet society or other Internet organizations, except as needed for the purpose of developing Internet standards in which case the procedures for copyrights defined in the Internet Standards process must be followed, or as required to translate it into languages other than English.

The limited permissions granted above are perpetual and will not be revoked by the Internet society or its successors or assigns.

This document and the information contained herein is provided on an "AS IS" basis and THE INTERNET SOCIETY AND THE INTERNET ENGINEERING TASK FORCE DISCLAIMS ALL WARRANTIES, EXPRESS OR IMPLIED, INCLUDING BUT NOT LIMITED TO ANY WARRANTY THAT THE USE OF THE INFORMATION HEREIN WILL NOT INFRINGE ANY RIGHTS OR ANY IMPLIED WARRANTIES OF MERCHANTABILITY OR FITNESS FOR A PARTICULAR PURPOSE.

Acknowledgement

Funding for the RFC Editor function is currently provided by the Internet society. 